\title{
Dairy Cattle Breeding Simulation Program: A simulation program to teach animal breeding principles and practices
}

\author{
J. F. Medrano, ${ }^{* 1}$ A. Ahmadi, ${ }^{*}$ and J. Casellas* $† \ddagger$ \\ *Department of Animal Science, University of California, Davis 95616 \\ †Genètica i Millora Animal, IRTA-Lleida, 25198 Lleida, Spain \\ ‡Departament de Ciència Animal i dels Aliments, Universitat Autònoma de Barcelona, 08193 Bellaterra, Spain
}

\section{ABSTRACT}

A dairy cattle breeding simulation program (DCBSP v.4.9) has been developed to teach undergraduate and graduate students animal breeding principles associated with selection for multiple traits in dairy cattle. The current version of the program was written in FORTRAN 90, and a web-based interface was developed for the students to interact with the program in the teaching environment. This software simulates a population of dairy cattle herds and artificial insemination bulls through several generations by integrating students' decisions about mating, culling, and selection of new heifers and bulls based on a multivariate animal mixed model evaluation and marker-assisted selection. All simulation parameters (e.g., number of herds and cows per herd, variance components, effect of genetic markers) can be defined by the administrator of the program in relation to the animal breeding course. During each running period, the program simulates the composition of each herd during a virtual year, generating new calves and new productive records and performing a genetic evaluation for all productive traits. A herd-specific productive summary of all demographic, productive, and genetic data is provided to the students at the end of each simulation period. After several running periods, the genetic trend can be evaluated, providing a realistic experience for the development of animal breeding skills that will be relevant to students with a basic knowledge of animal breeding. Earlier versions of this program have been used at several universities where it has proven to be a very useful teaching tool to illustrate the theoretical basis of animal breeding in livestock.

Key words: dairy cattle, selection, software, teaching

Received August 12, 2009.

Accepted January 26, 2010.

${ }^{1}$ Corresponding author: jfmedrano@ucdavis.edu

\section{INTRODUCTION}

Animal breeding courses typically focus on multiple theoretical concepts (e.g., Mendelian inheritance, polygenic variation, resemblance between relatives, response to selection, inbreeding) and a varying degree of statistical methodologies (e.g., ANOVA components, selection indices, mixed model evaluation, markerassisted selection) that cannot be easily demonstrated with real livestock. Hence, computer simulations have been used very successfully for teaching animal breeding and genetics in undergraduate and graduate courses (Heidhues and Henderson, 1961; Willham, 1968; Long et al., 1978).

Computer simulations provide multiple advantages for teaching animal breeding: 1) there is no economic cost to generate virtual animals, and only a small surcharge related to computational services is required; 2) time can be appropriately modeled to accommodate years or decades of real selection in shorter time units of virtual simulation (e.g., hours, days, or weeks); and 3) simulations can be repeated or moved backward and the decisions taken can be easily corrected and reanalyzed, avoiding the loss of opportunities and penalties from wrong decisions in real selection programs in livestock. Several simulation programs have been used for teaching animal breeding (Long et al., 1978; Hocking et al., 1983; Buchanan et al., 1988), and for the most part they focused on simplified population structures (e.g., a single beef cattle herd; Buchanan et al., 1988) or had limitations in terms of genetic evaluation methodologies (e.g., contemporary comparison indices; McGilliard and Edlund, 1979).

Our main objective in developing this simulation program was to provide a flexible program for teaching animal breeding based on a multi-herd dairy cattle framework allowing free interaction of students with their virtual herd through a web interface. The program we developed was an adaptation of the mainframe program, Dairy Cattle Breeding Simulation Program (DCBSP), written for these purposes by McGilliard and Edlund (1979). The current version (version 4.9) includes multivariate BLUP (Henderson, 1973) of the 
additive genetic merit for 4 dairy traits (milk yield, fat and protein percentages, and SCS). In addition, the program provides genotypic information and inbreeding coefficients to allow for integrated selection decisions involving selection indices, marker-assisted selection, and inbreeding depression. All of this information is summarized in detailed output files where the genetic trend can be easily evaluated, providing a realistic experience for the development of animal breeding skills that is relevant to students. As a whole, this software makes possible a direct interaction between a student and his or her own virtual herd, accommodating years of genetic selection into a few weeks of an undergraduate or graduate course. The active participation of the students during the simulation process increases student motivation, leading to greater interaction and information flow between instructors and students.

\section{PROGRAM DESCRIPTION}

\section{Background}

The DCBSP had its early origins in a simulation program written in FORTRAN code on punched cards by Lon D. McGilliard at the Department of Dairy Science at Michigan State University in the late 1950s. This program included a single trait (milk yield) and was used over several decades with the goal of 20 virtual generations in a 10-wk period (M. L. McGilliard, Virginia Tech University, Blacksburg; personal communication). In the mid-1970s, Mike McGilliard and Dennis Edlund developed the first multi-trait version of the DCBSP at the Department of Dairy Science at Virginia Tech University (Blacksburg), by combining the features of the early DCBSP and the SIMBULL program previously developed by Richard Willham and Gordon Thompson at Iowa State University. These efforts converged with the creation of the 1979 version of DCBSP (McGilliard and Edlund, 1979), which was upgraded with a microcomputer input interface by Ron Pearson at Virginia Tech University in the early 1990s. The program was distributed to 31 US universities as well as 18 universities overseas (M. L. McGilliard; personal communication). Over the decades, this software provided a useful experience for the development of animal breeding skills to animal breeding students. The 1979 version of DCBSP simulated up to 100 dairy cattle herds with up to 100 cows per herd during several virtual generations by integrating students' decisions about mating, culling, and selection of new heifers and AI bulls. Before each running period, a new set of heifers and yearling bulls was available for selection, and all individuals were genetically evaluated for several dairy-related traits by contemporary comparison methods (Robertson and
Rendel, 1954). Each running period simulated a virtual pseudo-year where all selected cows and heifers were mated, calved, and provided a new set of phenotypic records. At the University of California-Davis, Graham Gall implemented the program in the early 1980s and, as student enrollment increased in the animal science breeding course, the program went through several rounds of improvement to facilitate students' interaction with the herds and data. Recently, a web interface was developed for data input and output.

\section{Program Overview}

In 2006, DCBSP was completely rewritten under the FORTRAN 90 programming language. The executable file of this new version runs in DOS-Windows operating systems. The current version, DCBSP v.4.9, accommodates new statistical approaches (e.g., BLUP, markerassisted selection) and provides a more realistic framework within current methods for dairy cattle breeding. A schematic overview of the program's running process is presented in Figure 1. The first run of the DCBSP program simulates the breeding structure at a given date previously defined in the parameter file (Figure 2 ), with the desired number of herds and founder AI bulls and cows. For each herd, cows are simulated as founders without known ancestors or daughters of these founder cows and the AI founder bulls. Cows are simulated under a predefined age structure in the parameter file (see Ducrocq et al., 1988) and all productive records pertaining to previous lactations are stored for further genetic evaluations. Additionally, all calves born during the previous generation [i.e., in the previous 20 (males) and 14 (females) months] are stored as potential selection candidates. The DCBSP ends with the genetic evaluation of all bulls, cows, yearling bulls, heifers, and calves, and the generation of detailed summary files with all phenotypic and genetic data.

All of this information becomes available for the students, who must make appropriate decisions about AI bulls for the entire population and about cows and heifers from their own herd. The list of available AI bulls for the next year is chosen by a student committee by picking a combination of tested AI bulls and some new AI bulls from the list of available (or nominated) yearling bulls (male calves born 8 to 20 mo before the current virtual date). Then, each student must decide which cows and heifers (female calves born 2 to 14 mo before the current virtual date) are culled and which bull will mate with each cow or heifer during the next productive year according to the timing scheme shown in Figure 3. These decisions can be made based on the predicted breeding values of each animal, its genotype 


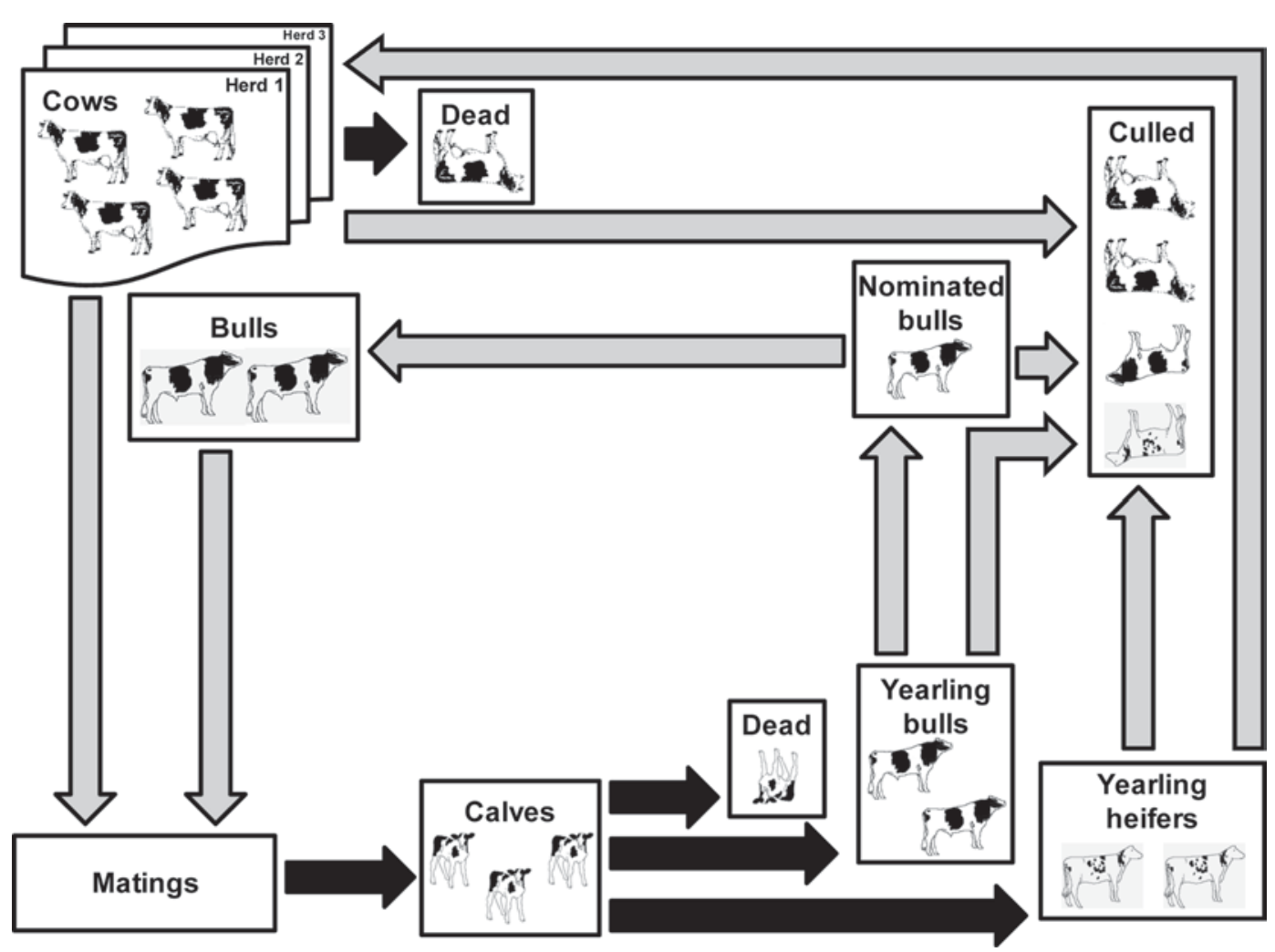

Figure 1. Description of the Dairy Cattle Breeding Simulation Program (DCBSP) running process. The gray arrows identify students' decisions, whereas the black arrows are part of the simulation processes. At the start of the simulation, each student is assigned his or her own individual herd consisting of cows, yearling heifers, yearling bulls, and calves. The student must decide which cows and heifers are culled and which bull will mate each cow or heifer for the next productive cycle. In each yearly cycle, calves are born and production records and genetic evaluation become available to students. Calves and cows can die in each herd according to predetermined probabilities. Following a pattern of virtual time, yearling bulls can be nominated to be selected as potential bulls that are then included in the mating scheme and progeny tested. All yearling bulls that are not selected for progeny testing are culled. Yearling heifers become cows if they are selected as cow replacements and mated in the next cycle. Yearling heifers and cows that are not mated are culled from the herd. The cycle of cow selection, bull nomination, and matings is typically repeated for 9 to 10 virtual years, but can be continued as desired.

for 2 biallelic genetic markers (MK1 and MK2), or inbreeding coefficient among other factors.

At this point, students' decisions are uploaded to the DCBSP and the program runs again to simulate matings, calvings, and productive records (Figure 1) for the next productive year (the simulation interval will be referred here as a virtual year), as well as to perform a new genetic evaluation and generate a new set of summary files. An appropriate interface has been developed to upload student decisions via the Internet.

\section{Simulation Processes}

Reproductive and Productive Cycle. Although the DCBSP summarizes each individual and herd at the end of the virtual year, the program simulates all reproductive (i.e., AI, calving) and productive events (i.e., lactation beginning and end, death) for each cow and calf on a sequential time frame as described in Figure 3. The time intervals for productive and repro- ductive processes were fixed to reasonable values for a typical US dairy production environment. After birth, selected female calves are maintained in the herd for $425 \mathrm{~d}$ ( $\sim 14 \mathrm{mo})$ before the first mating. Note that cow fertility is determined by the program administrator in the parameter file (Figure 2, element 17) and, after AI, cows can become pregnant or remain open. Open cows are inseminated again $21 \mathrm{~d}$ later (the cow is considered "open" between calving and the successful AI). Calving occurs after $275 \mathrm{~d}(\sim 9 \mathrm{mo})$ of gestation and only one new calf is generated with a $50 \%$ probability for each sex (twin births are not allowed). The cow is inseminated again $60 \mathrm{~d}$ after calving. The lactation period spans $305 \mathrm{~d}(\sim 10 \mathrm{mo})$ and the new set of phenotypic records for dairy traits is stored for genetic evaluation purposes at the end of the lactation. This productive cycle generates a continuous uploading of new calves and lactations, mainly determined by the birth date of each cow and its fertility performance. Moreover, the birth date of a given cow is determined by the repro- 
Parameter file

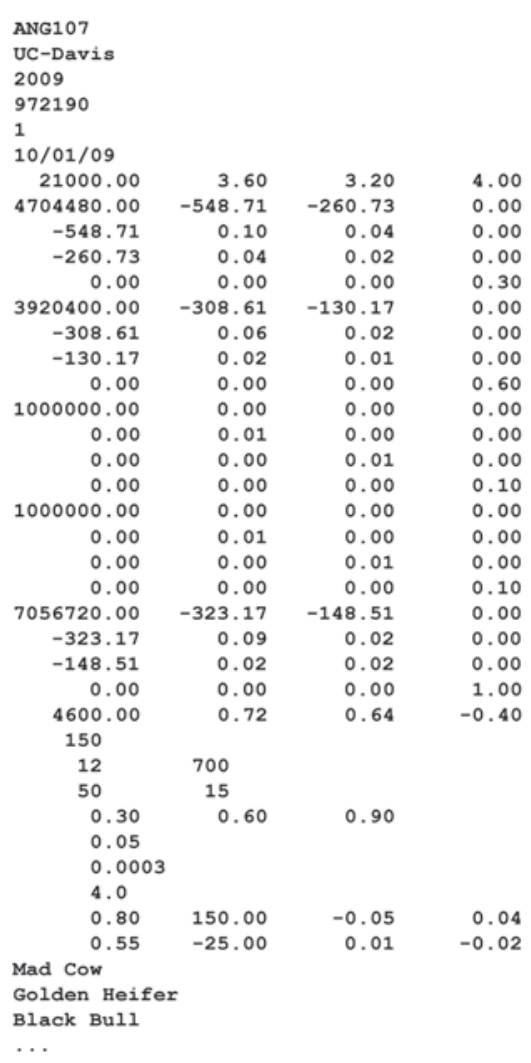

Elements of the file

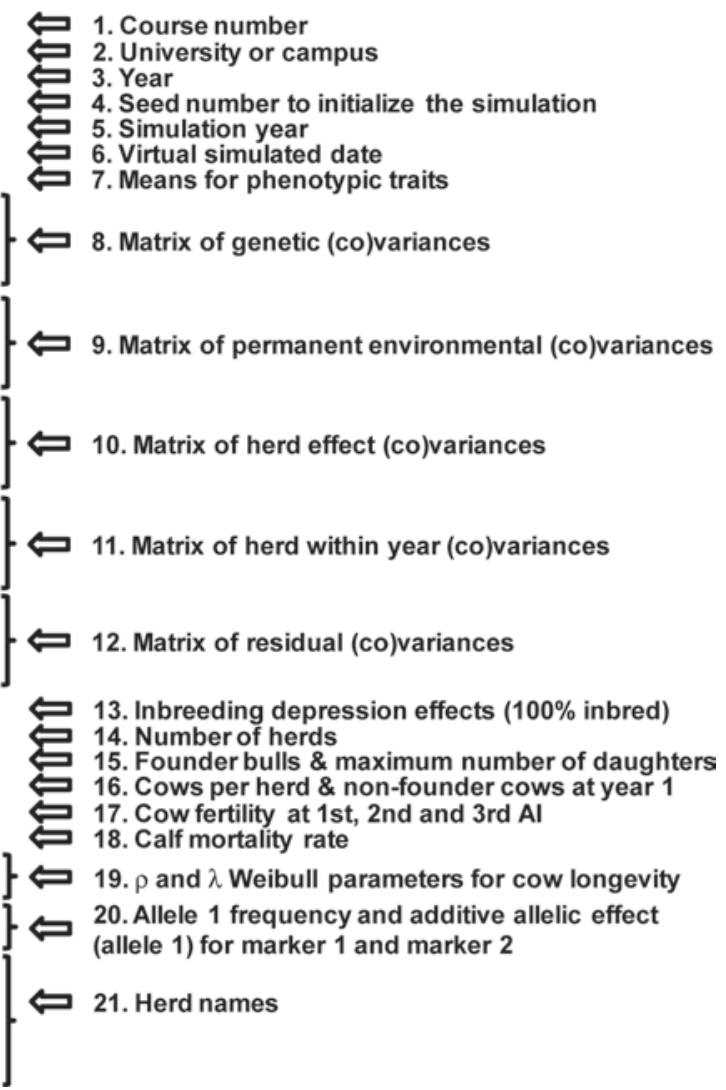

Figure 2. Example of the parameter file for the Dairy Cattle Breeding Simulation Program (DCBSP) and summary of the elements.

ductive sequence of its mother and so on. Note that all these processes occur within the virtual years of the program.

Initial Population. The DCBSP has been written on a highly flexible structure with a maximum of 1,000 herds and 99 cows per herd to accommodate a wide range of animal breeding course requirements. The initial population is defined by the program administrator in the parameter file by establishing the desired number of herds, cows per herd, and founder bulls (Figure

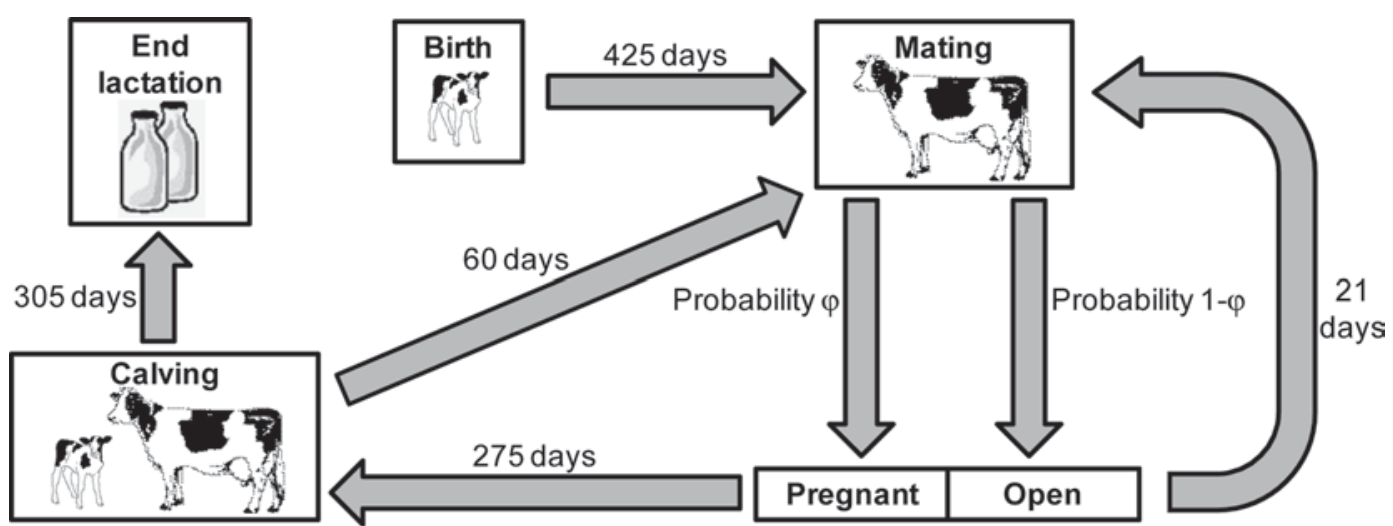

Figure 3. Diagram showing the sequential timeframe of the reproductive and productive cycles simulated by the Dairy Cattle Breeding Simulation Program (DCBSP). The age of founder cows is sampled from a Weibull distribution and prior lactations to the virtual start date are simulated and stored. The probability of becoming pregnant $(\varphi)$ is defined in the parameter file (Figure 2 , element 17 ). 
2, elements 14 to 16). At the beginning, the DCBSP simulates all bulls and several cows as founders without known ancestors, whereas the remaining cows are generated as offspring from founder bulls and cows (see Figure 2, element 16). Note that all the reproductive and productive history of initial cows before the virtual starting date (Figure 2, element 6) is simulated and stored following the process shown in Figure 3. These preliminary data allow for the genetic evaluation of the founder bulls (from daughters with lactation records) and cows at the beginning of the simulation.

Aging and Mortality Processes for Cows, Bulls, and Calves. Following Ducrocq et al. (1988), the aging process for cows is modeled under a Weibull distribution with parameters $\rho$ and $\lambda$ (Figure 2, element 19). The length of the productive life ( $l$ ) for cows born during the simulation process is generated by the transformation method (Press et al., 1992) from

$$
l=\left[-\frac{\ln (1-u)}{e^{\rho \ln (\lambda)}}\right]^{\frac{1}{\rho}}
$$

where $u$ is a random number sampled from a uniform distribution between 0 and 1 . Two longevity values are required for founder cows, current age at the beginning of the simulation $\left(l_{c}\right)$ and overall length of productive life $\left(l^{\prime} ; l^{\prime}>l_{c}\right)$. Both values are obtained from equation [1] although, in this case, $u$ is a random sample from a uniform distribution between 0 and 1 for $l_{c}\left(u_{c}\right)$ and a random sample from a uniform distribution between $u_{c}$ and 1 for $l^{\prime}$.

Longevity is not properly defined for bulls because semen doses can be frozen and used long after bull's death. To discourage extensive use of the best bulls, bulls are identified as having "died" in the output file when a maximum number of daughters in production is reached in the whole population (Figure 2, element 15). Nevertheless, this is only an operational formality and the DCBSP allows inseminating cows with semen from these bulls if required. For calves, the mortality rate is specified in the parameter file (Figure 2, element 18) and it occurs at random among the calves.

Animal-Specific Simulations. The DCBSP program stores all parental relationships during the simulation process. For each individual, real genetic and permanent environmental effects are generated and stored at the beginning of the simulation or at birth. The additive genetic effect for a founder individual $\left(\mathbf{a}_{j}\right)$ is sampled from a multivariate normal distribution $\mathbf{a}_{\text {}}$ $\sim \operatorname{MVN}(\mathbf{0}, \mathbf{G}), \mathbf{0}$ being a $4 \times 1$ vector of zeros and $\mathbf{G}$ being the $4 \times 4$ matrix of genetic (co)variances speci- fied in the parameter file (Figure 2, element 8). For nonfounder individuals, $\mathbf{a}_{j}$ is simulated as

$$
\mathbf{a}_{j}=\frac{1}{2}\left(\mathbf{a}_{\text {sire }}+\mathbf{a}_{d a m}\right)+\varphi_{j}
$$

where $\mathbf{a}_{\text {sire }}$ and $\mathbf{a}_{d a m}$ are the additive genetic effects for the parents of the $j$ th individual, and $\varphi_{j}$ is the $4 \times 1$ vector of Mendelian sampling terms generated from

$$
\boldsymbol{\varphi}_{j} \sim M V N\left(\mathbf{0}, \frac{1}{2}\left(1-\frac{1}{2}\left(f_{\text {sire }}+f_{\text {dam }}\right)\right) \mathbf{G}\right) .
$$

Note that $f_{\text {sire }}$ and $f_{\text {dam }}$ are the inbreeding coefficients of $j$ 's parents calculated as by Quaas (1976). The permanent environmental effect $\left(\mathbf{p}_{j}\right)$ is sampled from $\mathbf{p}_{j} \sim$ $\operatorname{MVN}(\mathbf{0}, \mathbf{P})$, where $\mathbf{P}$ is the $4 \times 4$ matrix of permanent (co)variances (Figure 2, element 9).

The genotype for 2 independent biallelic markers is simulated under a standard Mendelian inheritance process. For founder individuals, the allelic frequency of allele $1\left(\phi_{1}\right)$ is specified in the parameter file (Figure 2, element 20; first value of each row), and genotypes 11, 12 , and 22 are sampled at random with probabilities $\phi_{1}^{2}, 2 \phi_{1}\left(1-\phi_{1}\right)$, and $\left(1-\phi_{1}\right)^{2}$, respectively. The genotype for nonfounder individuals is generated at random by picking one allele from each parent's genotype.

Lactation Records. For the ith lactation of the $j$ th cow, the DCBSP samples the phenotypes for milk yield (MILK), fat percentage (\%FAT), protein percentage (\%PROT), and SCS (SC) from a multivariate normal distribution

$$
\mathbf{y}_{i j} \sim M V N\left(\boldsymbol{\mu}+\mathbf{X}_{i j} \boldsymbol{\beta}+\mathbf{Z}_{i j} \mathbf{p}_{j}+\mathbf{Z}_{i j} \mathbf{a}_{j}, \mathbf{R}\right),
$$

where $\mathbf{y}_{i j}$ is the $4 \times 1$ vector of phenotypes influenced by the overall means $(\boldsymbol{\mu}$; dimension $4 \times 1$; Figure 2 , element 7), systematic effects ( $\boldsymbol{\beta}$; dimension $4 \times 1$ ), and the permanent and the additive genetic contribution of the $j$ th cow; $\mathbf{X}_{i j}$ and $\mathbf{Z}_{i j}$ are appropriate incidence matrices and $\mathbf{R}$ is the $4 \times 4$ matrix of residual (co)variances (Figure 2, element 12). Systematic effects include 2 environmental factors, herd $\left(\mathbf{h}_{i}\right.$; dimension $\left.4 \times 1\right)$ and herd within year $\left[\mathbf{h}\left(\boldsymbol{\psi}_{l}\right)\right.$; dimension $\left.4 \times 1\right]$, and 3 genetic contributions of the $j$ th cow accounting for inbreeding depression (i; dimension $4 \times 1$ ), additive allelic effect of MK1 $\left(\mathbf{m}_{1}\right.$; dimension $4 \times 1$; Figure 2, element 20) and additive allelic effect of MK2 $\left(\mathbf{m}_{2}\right.$; dimension $4 \times$ $1)$. Both $\mathbf{h}_{l}$ and $\mathbf{h}\left(\boldsymbol{\psi}_{l}\right)$ effects for the lth virtual year of simulation are sampled from $\mathbf{h}_{l} \sim \operatorname{MVN}(\mathbf{0}, \mathbf{H})$ and $\mathbf{h}\left(\boldsymbol{\psi}_{l}\right) \sim \operatorname{MVN}(\mathbf{0}, \boldsymbol{\psi})$, respectively, where $\mathbf{H}$ and $\boldsymbol{\psi}$ 
are appropriate $4 \times 4$ (co)variance matrices (Figure 2, elements 10 and 11, respectively). Although herd and herd-within-year effects could be simulated under other distributions, the multivariate normal process allows a straightforward definition of its magnitude (variance) as well as accounting for relationships between traits (covariances) with minimum parameterization. The 3 remaining systematic effects are modeled as continuous covariates characterized by the linear effect of $f_{j}(\mathbf{i}$; Figure 2, element 13) or by the standard -1 (genotype 11), 0 (genotype 12), and 1 (genotype 22) $\left(\mathbf{m}_{1}\right.$ and $\left.\mathbf{m}_{2}\right)$ parameterization described by Falconer and Mackay (1996).

\section{Genetic Evaluation Through a Multivariate Animal Linear Mixed Model}

At the end of the simulation process, a new multiherd genetic evaluation of all individuals included in the pedigree is performed by fitting a multivariate animal linear mixed model using BLUP methodologies (Henderson, 1973). Assuming $n$ phenotypic records for each trait and $m$ individuals in the pedigree, the analytical model fit was the one assumed during the simulation process:

$$
\mathbf{y}^{\bullet}=\mathbf{X}^{\bullet} \boldsymbol{\beta}^{\bullet}+\mathbf{Z}^{\bullet} \mathbf{p}^{\bullet}+\mathbf{Z}^{\bullet} \mathbf{a}^{\bullet}+\mathbf{e}^{\bullet},
$$

where $\mathbf{y}^{\bullet}$ is the $(4 n) \times 1$ vector of phenotypic records sorted within lactation, $\boldsymbol{\beta}^{\bullet}$ is the vector of systematic effects, $\mathbf{p}^{\bullet}$ is the vector of permanent environmental effects, $\mathbf{a}^{\bullet}$ is the vector of additive genetic effects, $\mathbf{e}^{\bullet}$ is the vector of residuals, and $\mathbf{X}^{\bullet}$ and $\mathbf{Z}^{\bullet}$ are appropriate incidence matrices. Phenotypes are assumed to be sampled from a multivariate normal distribution $\mathbf{y}^{\bullet} \sim M V N\left(\mathbf{X}^{\bullet} \boldsymbol{\beta}^{\bullet}+\mathbf{Z}^{\bullet} \mathbf{p}^{\bullet}+\mathbf{Z}^{\bullet} \mathbf{a}^{\bullet}, \mathbf{R}^{\bullet}\right)$ with residual (co) variance matrix $\mathbf{R}^{\bullet}=\bigoplus_{o=1}^{n} \mathbf{R}$. Both $\mathbf{p}^{\bullet}$ and $\mathbf{a}^{\bullet}$ are $(4 m) \times 1$ sorted by trait within individual and modeled under the following multivariate normal processes, $\mathbf{p}^{\bullet} \sim M V N\left(\mathbf{0}, \mathbf{P}^{\bullet}\right)$ and $\mathbf{a}^{\bullet} \sim M V N\left(\mathbf{0}, \mathbf{G}^{\bullet}\right)$, with $\mathbf{P}^{\bullet}=\mathbf{I} \otimes \mathbf{P}$ and $\mathbf{G}^{\bullet}=\mathbf{A} \otimes \mathbf{G}$, respectively. Note that $\mathbf{I}$ is an $m \times m$ identity matrix, $\mathbf{A}$ is the numerator relationship matrix between individuals (Henderson, 1976; Quaas, 1976), and $\otimes$ and $\oplus$ designate the Kronecker product and sum, respectively. True simulation matrices $\mathbf{R}, \mathbf{P}$, and $\mathbf{G}$ are assumed during the genetic evaluation. This mixed animal model is iteratively solved by the GaussSeidel approach (Misztal et al., 1987). Predicted breeding values for calves are calculated as the average of the predicted breeding value of their parents.

\section{Output Files}

At the end of each running period, DCBSP provides a full summary of all reproductive, productive, and genetic data. The output files provide a detailed characterization at the animal, herd, and population levels.

Herd File. This is a space-delimited file provided for every herd and subdivided in 4 sections. Section 1 provides information for each cow and heifers selected during the previous virtual year, on birth date and age, reproductive summary, productive summary, and status of the cow at the current virtual date. Section 2 focuses on the genetic information of the same cows and heifers, characterizing their ancestors up to the corresponding grandparents if available, breeding values (and reliability) for MILK, FAT\%, PROT\%, and SC in terms of PTA, genotype for MK1 and MK2, and inbreeding coefficient. Section 3 provides the same genetic information for yearling bulls (male calves born 8 to 20 mo before the current virtual date) and heifers (female calves born 2 to 14 mo before the current virtual date), and for the youngest male (born during the last $8 \mathrm{mo}$ ) and female (born during the last $2 \mathrm{mo}$ ) calves (data not shown). Section 4 provides the simulated and predicted values for the population mean, herd effect, and herd-within-year effect, as well as the average value for genetic, permanent environmental, and residual effects. In addition to this file, 2 more comma-delimited files are provided with the PTA of the 4 phenotypic traits for cows/heifers and yearling bulls, respectively, to ease the student's management of the genetic information with Microsoft Excel (Microsoft Corp., Redmond, WA) or similar software.

Herds Summary and AI Bull Summary File. This is a space-delimited file with 2 main sections. The first section provides a list of all herds in the simulation with the current number of cows, the average value for the phenotypic dairy traits from the lactations finishing during the last virtual year, the average PTA and corrected PTA (PTA plus the contributions of MK1, MK2, and inbreeding) for MILK, FAT\%, PROT\%, and SC, and allelic frequencies for MK1 and MK2, and average inbreeding. The second section provides the genetic information for all AI bulls.

Herd and Population Performance Files. These 2 files show the same structure but summarizing the performance of a given herd or the whole population across years. At the end of the simulation process, these files are provided to the students to characterize the phenotypic and genetic (Figures 4 and 5) trends across virtual years.

Bulls Summary File. The genetic information of all AI bulls is provided in a comma-delimited file with both simulated $\left(\mathbf{a}_{j} / 2\right)$ and predicted PTA for MILK, 

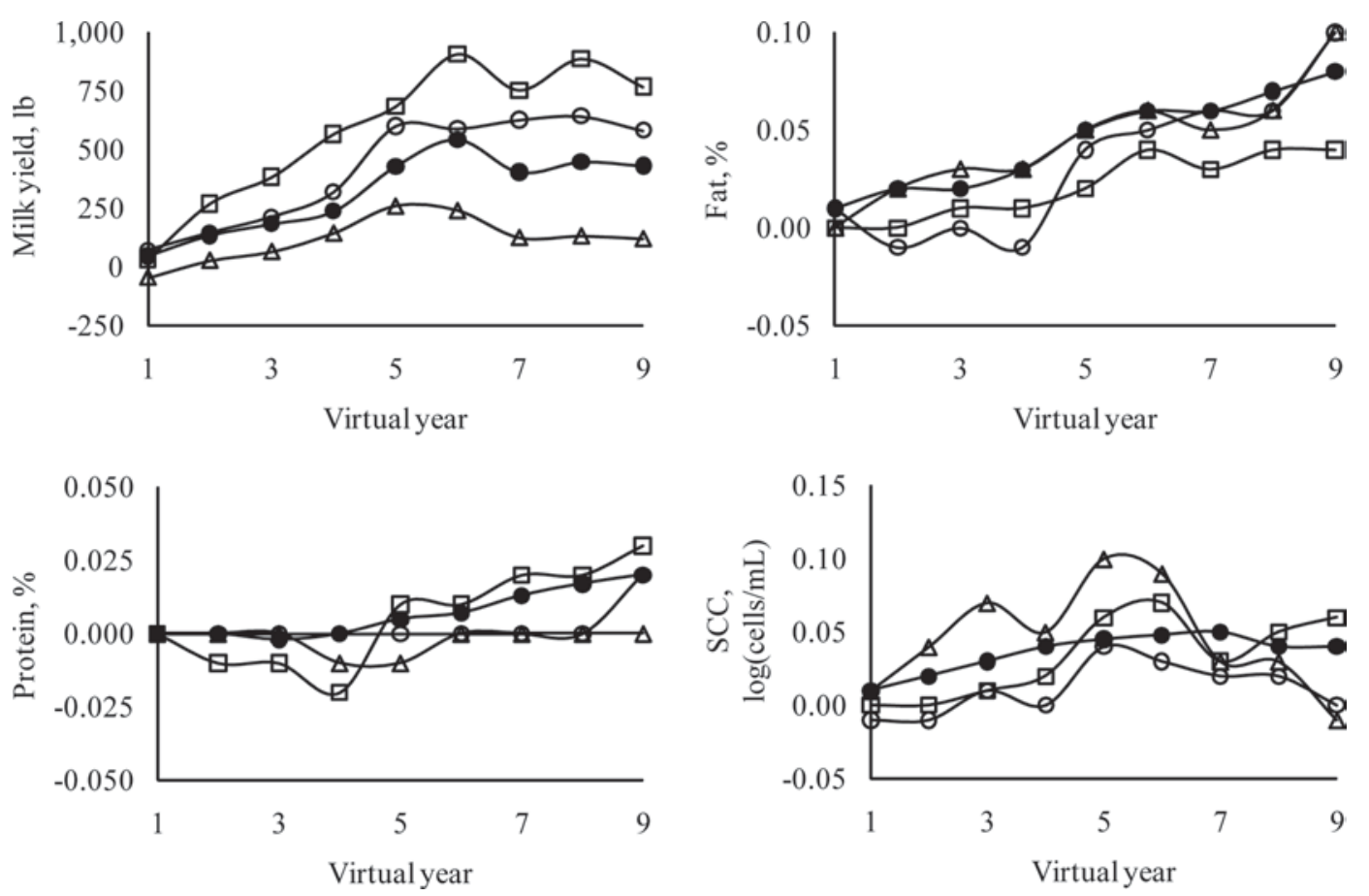

Figure 4. Genetic trends for the 4 dairy traits across 9 virtual years. The plots show the trend for a whole population of 150 herds $(\sim 8,000$ cows; - ) and 3 randomly picked herds (open symbols) selected for milk yield and fat percentage $(\bigcirc, \Delta)$ and for milk yield and protein percentage $(\square)$.
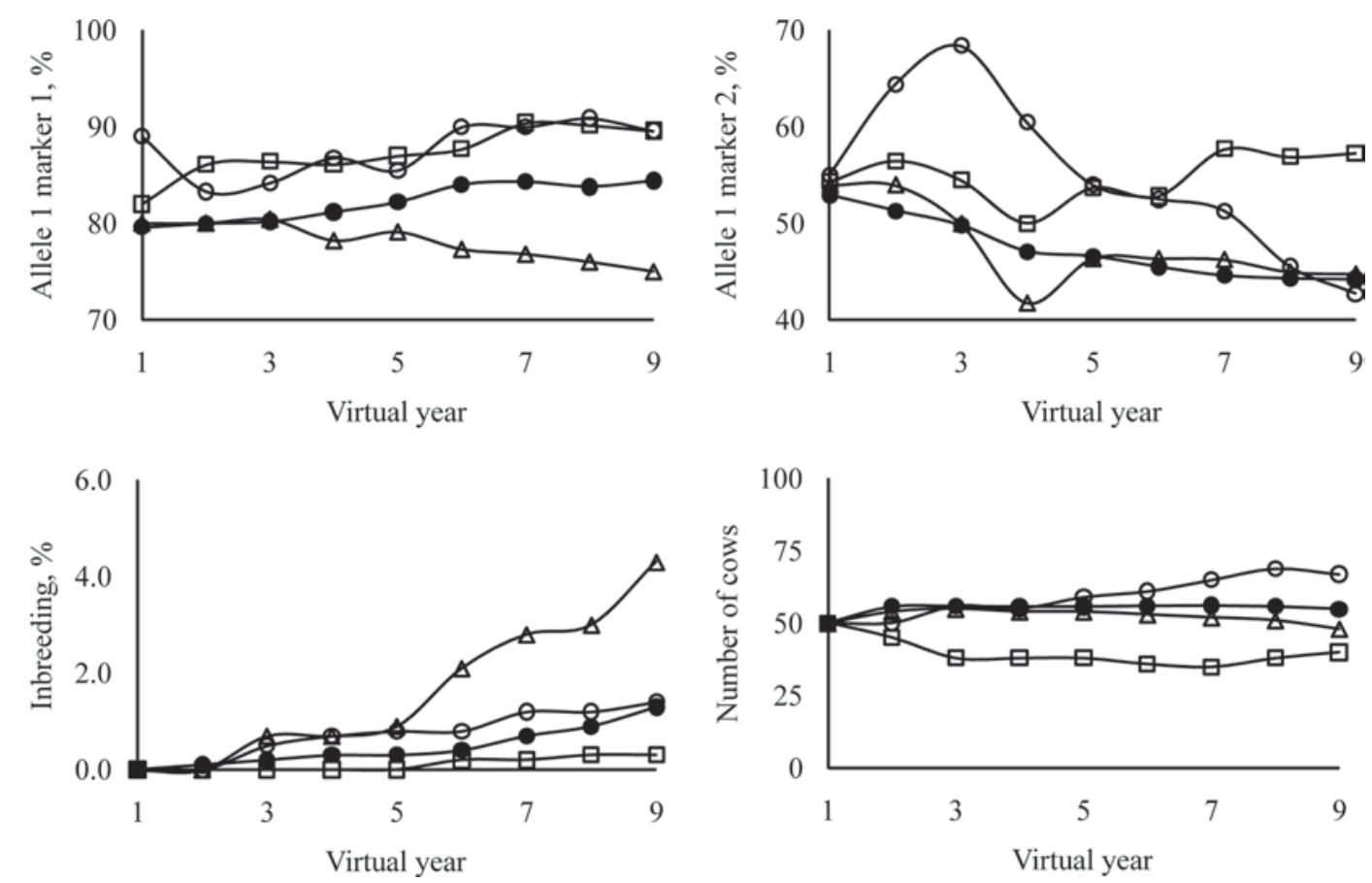

Figure 5. Trends for the allele frequency of genetic markers MK1 and MK2 (highest allele frequency in the founder population), inbreeding, and number of cows (specific herds) or average number of cows per herd (whole population). Plots for the whole population (•) and 3 randomly picked herds $(\square, \bigcirc, \Delta)$ were provided. Population of 150 herds $\sim 8,000$ cows. 


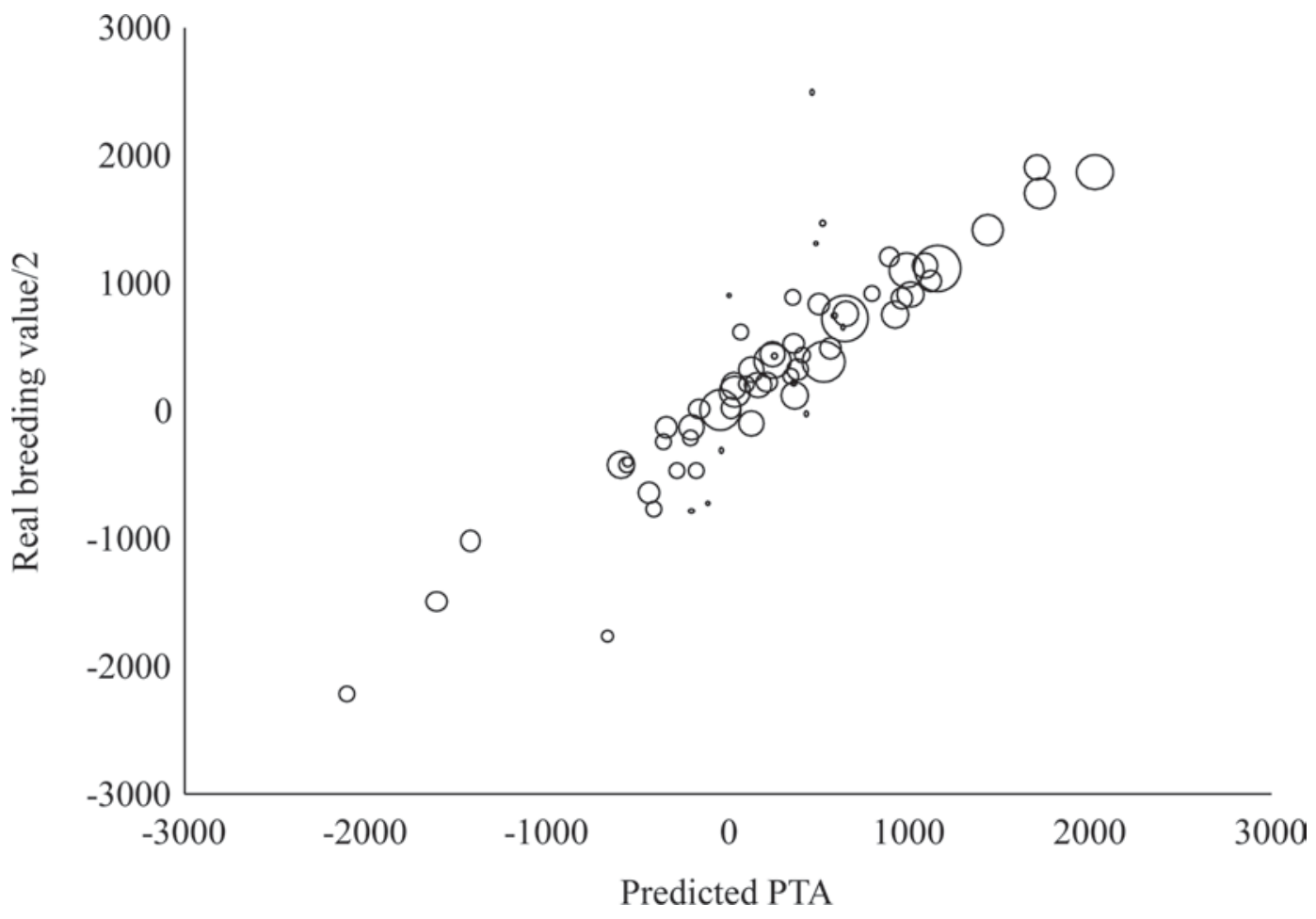

Figure 6. Real breeding value and predicted PTA for milk yield for AI bulls. The diameter of each spot characterized the number of evaluated daughters (this parameter ranged from 0 to 798$)$. Population of 150 herds ( 8,000 cows), 9 virtual years, and 85,000 lactations at the end of the simulation process.

FAT\%, PROT\%, and SC (Figure 6). Additional information about their genotype, inbreeding, parents, and grandparents, daughters in production, and average number of lactations per daughter is also provided.

\section{Web-Based Interface for Student-DCBSP Interaction}

The web interface was designed based on client/ server architecture for accessing online database tables. The server component consists of Microsoft Internet Information Services version 6.0 running on Microsoft Windows Server 2003 (Microsoft Corp.). The online database component is a Microsoft Access database with 5 tables: cows, bulls, mating, nomination, and students (Figure 7). The cows and bulls tables contain information on all the animals in each herd and the population as a whole. The mating table contains the pairs chosen to mate in each herd. The nomination table contains the bulls that are nominated weekly by the students. The users' tables contain the students name, herd number, and password for each student's account. The students provide the mating and nominating input through the Microsoft Web Browser client. The web pages were designed using the active server page (ASP) technology. Active server page is a special kind of HTML page with dynamic content that comes from the Access database.
The codes inside ASP are written in Visual Basic for Application, and commands in Structured Query Language are used to query or update the Access database.

The entire simulation system is managed by a scheduler, written in $\mathrm{C}++$ programming language. The scheduler runs twice a week, 1) for loading the tables and opening the system for mating, and 2) for running the simulation engine and generating output files. The scheduler manages the following 4 phases: nomination phase, loading phase, mating phase, and running phase. For the nomination phase, the scheduler opens the system for yearling bull nomination and the students have full access to the output files using the web interface. The nomination information is kept in the nomination table (Figure 7) to generate a list of all nominated bulls to be used by the student committee for selecting a set of bulls for the next run. After that, the scheduler loads 2 text files onto the bulls and cows tables (bulls.csv and cows.csv, respectively) on a predetermined day and time (loading phase). The bulls.csv file specifies the yearling and AI bulls selected by the students and is provided by the program administrator. The cows.csv file is automatically generated by the previous run of the DCBSP. The mating phase starts when the scheduler opens the system for the students 


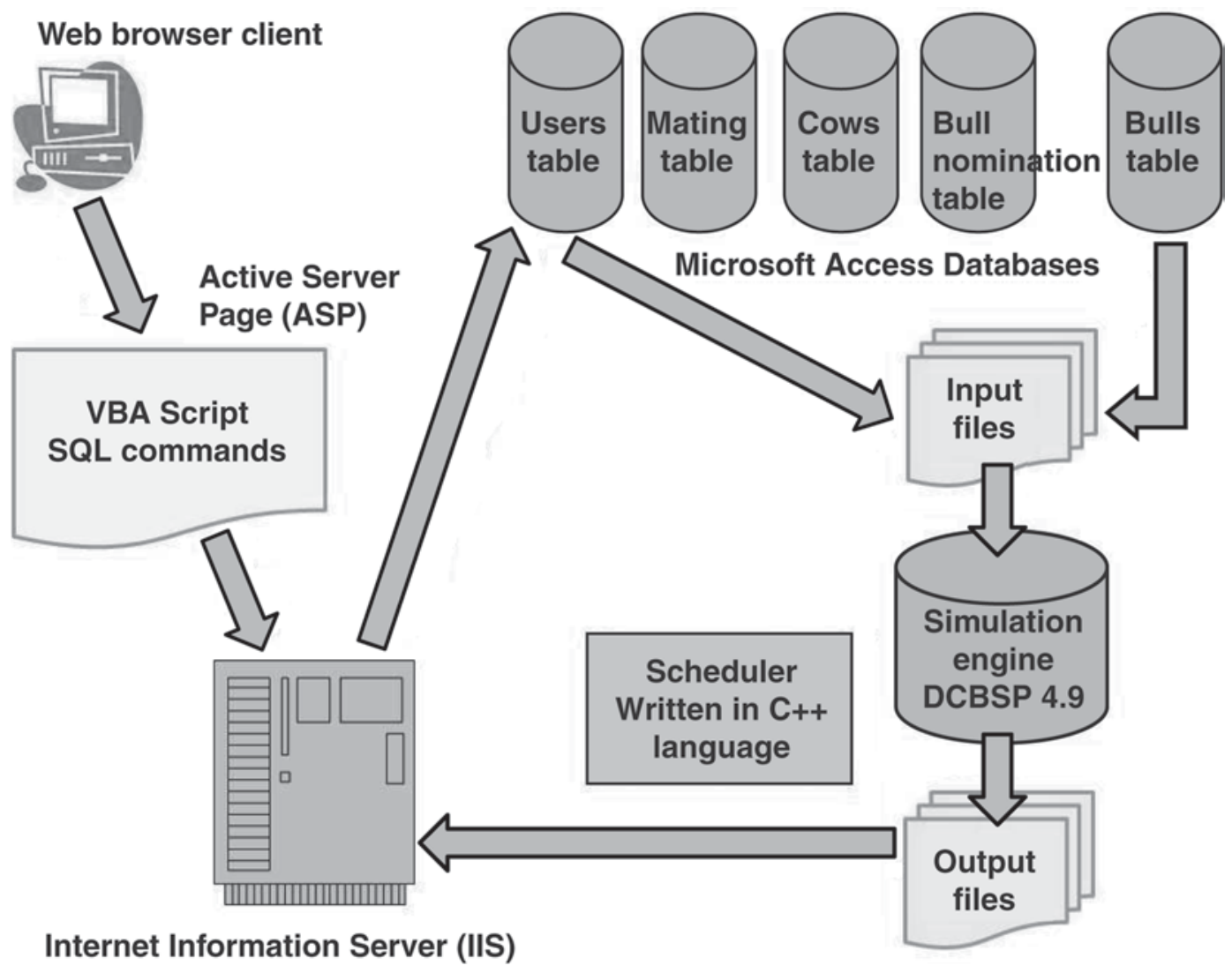

Figure 7. Diagram of the client/server architecture to manage the DCBSP computer simulation interface. The section to the left of the figure represents the front-end dynamic Web interface (wide arrows). This section includes the Web browser client where students access the simulation through an active server page that populates and obtains information from the Microsoft Access database tables. The section to the right of the figure represents the middleware scheduler that runs the simulation on a predetermined time schedule conforming to the needs of the class. The scheduler provides input files from the cows and bulls tables to the simulation engine (Dairy Cattle Breeding Simulation Program, DCBSP), collects the generated output files that are placed on the Web server for student access, and compiles the necessary output files for the next run of the simulation.

to complete their matings using the web browser. Each student has his or her own herd identified by the herd number and a password ( 4 digits) to $\log$ on to the web interface. At the end of mating period the scheduler exports the mating information from the mating table into a text file (matings.txt) and runs the DCBSP using matings.txt as input file (running phase). After the running phase, the scheduler collects the output files from the DCBSP and places them onto the web server for student access.

\section{Decisions to be Made by the Students at the Beginning of Each Virtual Year}

Nomination of Yearling Bulls. Immature bulls are distributed into 2 categories in the herd file: "yearling bulls" ( 8 to 20 mo old male calves available as potential AI bulls for the next virtual year) and "male calves" (less than 8 mo old calves, too young to be used as AI bulls during the next virtual year). Each student can nominate up to 3 of the yearling bulls as potential candidates to become AI bulls. This information is uploaded to the server by the webpage interface, whereas the un-nominated yearling bulls are assumed to be culled without further consideration. Note that the male calves are stored by the DCBSP and upgraded to yearling bulls at the end of the next virtual year.

Selection of New AI Bulls and List of Available Bulls for Mating. At the end of the nomination process, a list of several dozens or hundreds of nominated yearling bulls is available for the final selection of new AI bulls. This decision can be taken by the program administrator or by a student committee to select a few yearling bulls with high genetic merit to be progeny tested during the next mating seasons. The list of AI bulls for the next virtual year can be composed by all available bulls (founder bulls, yearling bulls selected during previous years, and currently selected yearling bulls) or restricted to a reduced number of animals selected by the program administrator or the student 
committee. This information is uploaded to the server by the program administrator and becomes available to the students for the next cycle of matings.

Selection/Culling and Mating of Heifers and Cows. Selection and culling decisions are taken once per virtual year and each student is responsible for his or her own herd. Immature females are distributed into "female calves" (less than 2 mo of age) and "heifers" ( 2 to 14 mo of age; they will be inseminated for the first time during the next virtual year), and selection/ culling decisions are made based on adult cows and heifers. Selected cows and heifers are mated in the web interface, and unmated cows and heifers are assumed to be culled.

\section{DISCUSSION}

Animal breeding and genetics relies on a series of theoretical and statistical principles that cannot be easily demonstrated on real livestock in undergraduate and graduate courses because of the obvious economic and time limitations. Therefore, the development of new simulation software for teaching animal breeding and genetics represents an important contribution.

Programs such as DCBSP make possible a direct interaction between students and their own virtual herds, allowing them to review their skills and ask questions on the animal breeding principles discussed in class. The results of years of genetic selection within a multi-herd scenario can be virtually accommodated into the few weeks of an undergraduate or graduate course, providing an accurate picture of the challenges and difficulties of a selection program in livestock species.

The DCBSP program builds a realistic scenario with multiple herds linked by a set of AI bulls comparable to the actual dairy breeding and production system that prevails worldwide. After the simulation of the founder individuals at the beginning of the multi-herd population, all additional cows and AI bulls are generated from this population, creating a complex network of across-herd genetic relationships because of the selection and mating decisions of each student within his or her own virtual herd. Moreover, the simulation design shown in Figure 3 creates a continuous structure of matings, calvings, and lactations distributed throughout the virtual year; it determines the availability of new heifers and yearling bulls for each herd, as well as the inclusion of new lactation records for the genetic evaluation process. Given that DCBSP focuses on the genetic aspects of dairy production, this software provides an accurate description of several genetic sources of variation and allows for different selection objectives depending on the objective and complexity of each course. This genetic information includes i) PTA for 4 phenotypic dairy traits, ii) genotype for MK1 and MK2, and iii) inbreeding coefficients. Hence, several selection objectives can be suggested; for example, marker-assisted selection on MK1, MK2, or both; genetic selection based on PTA from a single trait, 2 or more traits under positive genetic covariances, or 2 or more traits under negative genetic covariances; and genetic selection of one or more traits with restrictions for the increase of inbreeding or the frequency of negative alleles for MK1 and MK2. This flexibility in the selection objectives, combined with the definition of the simulation parameters by the program administrator, makes the DCBSP software a very useful and powerful tool for teaching animal breeding.

The DCBSP program runs in a very simple environment in which only a few space-delimited files are required as input files, and the program provides several space-delimited files and comma-delimited files as output files. This allows for 2 different implementations of DCBSP, depending on whether the webpage interface is used or not. If used, each student interacts with the DCBSP program through his or her own webpage interface, which allows for a straightforward uploading of the student's mating decisions (and nominations for new AI bulls) to the server. After each running process, all output files are available for each student in the respective webpage account, making it very user friendly for undergraduates. The responsibilities of the program administration are reduced to uploading the new AI bulls and the teaching supervision of the process. Without the webpage interface, the program administrator must interact with the students to integrate their decisions about AI bulls and matings, generate all the required input files, provide them to the DCBSP, and submit the appropriate files to the students. This second approach requires dedicated management of the simulation. In any case, DCBSP can be adjusted to the requirements of the different users, creating an appropriate application for each situation.

This simulation program is a good complement to a sound animal breeding course, providing the means for putting these principles to work. The active participation of the students during the simulation process increases students' motivation due to i) the complexity of making selection decisions using several sources of information and traits, ii) the generation of new questions when the students confront the practical aspects of animal breeding, and iii) the quick development of an intense and motivating competition between students. The performance of the selection and mating decisions taken by each student can be easily compared with the genetic progress of classmates' herds in the herds summary file, creating competition and motivation to take the right decisions in the next selection and mating sce- 
nario. This additional motivation generates questions around the properties of the genetic evaluation of the animals and the practical aspects of animal selection, leading to greater interaction and information flow between instructors and students.

This program has been used in animal breeding courses at several universities. The program has numerous error-checking routines and is designed so that students and instructors can focus on the breeding principles illustrated by the program, rather than on computer programming issues. Users with varying experience with animal breeding and with dairy cattle have found it educational because it is easy to use and progress is made quickly. The webpage interface and the program are hosted at the Department of Animal Science of the University of California-Davis and can be implemented for specific courses. Questions on the necessary arrangements and availability should be submitted to the corresponding author of this manuscript.

\section{ACKNOWLEDGMENTS}

The development of this software was funded by a University of California, Davis Undergraduate Institutional Improvement Minigrant U03-503SP and by the California Agricultural Experimental Station. The research contract of J. Casellas was partially financed by Spain's Ministerio de Ciencia e Innovación (programs Juan de la Cierva and José Castillejo). The authors acknowledge the efforts of Graham A. E. Gall and Curt M. Finley for the improvement of early versions of the DCBSP package, and Ricardo A. Verdugo for an earlier program to create the mating file (all of University of California, Davis). The final testing of the DCBSP v.4.9 would have been impossible without the collaboration of the students from the animal science undergraduate Animal Breeding course at the University of CaliforniaDavis, our colleagues at the University of California, Davis (R. J. Gularte, J. Chitwood, A. Islas-Trejo, R. Lathan, G. Rincón, E. Robb and S. Wickramasinghe), the New Mexico State University (M. G. Thomas), the University Autònoma of Barcelona, Spain (M. Amills,
R. Bach, G. Caja, J. Estellé, M. Fina, E. Flores, J. Piedrafita, N. Sastre, M. Salinas, and A. Tomás), the Agrifood Research and Technology Institute, Spain (M. Martínez-Giner and L. Varona), and several nonacademic friends (P. Alonso, D. Casellas, R. Salgas, and D. Vidal).

\section{REFERENCES}

Buchanan, D. S., L. G. Burditt, and R. L. Willham. 1988. Cowgame A beef-cattle selection simulation program. J. Hered. 79:215.

Ducrocq, V., R. L. Quaas, E. J. Pollak, and G. Casella. 1988. Length of productive life of dairy cows. 1. Justification of a Weibull model. J. Dairy Sci. 71:3061-3070.

Falconer, D. S., and T. F. C. Mackay. 1996. Introduction to Quantitative Genetics. Longmans Green, Harlow Essex, UK.

Heidhues, T., and C. R. Henderson. 1961. Teaching selection principles with herd records generated by an electronic computer. J. Anim. Sci. 20:659-664.

Henderson, C. R. 1973. Sire evaluations and genetic trends. Pages 1041 in Animal Breeding and Genetics Symposium in Honor of Dr. Jay L. Lush. American Society of Animal Science and American Dairy Science Association, Champaign, IL.

Henderson, C. R. 1976. Simple method for computing inverse of a numerator relationship matrix used in prediction of breeding values. Biometrics 32:69-83.

Hocking, P. M., J. L. Foulley, P. H. Petersen, H. Schulte-Coerne, and A. Zarnecki. 1983. Computer programs for teaching animal breeding and genetics. Livest. Prod. Sci. 10:589-599.

Long, C. R., K. H. Maddy, J. W. Clawson, H. W. Newland, R. R. Shrode, and J. W. Oxley. 1978. An inventory of computer programs used in animal science teaching, research and extension. Pages 38-64 in Symposium on Use of the Computer in Animal Science Teaching, Research and Extension. American Society of Animal Science, Champaign, IL.

McGilliard, M. L., and D. Edlund. 1979. Dairy cattle breeding simulation program. Virginia Polytechnic and State University, Blacksburg.

Misztal, I., D. Gianola, and L. R. Schaeffer. 1987. Exploration and convergence criteria with Jacobi and Gauss-Seidel iteration in animal models. J. Dairy Sci. 70:2577-2584.

Press, W. H., S. A. Teukolsky, W. T. Vetterling, and B. P. Flannery. 1992. Numerical Recipes in Fortran 77. The Art of Scientific Computing. Vol. 1, 2nd ed. Cambridge University Press, Cambridge, UK.

Quaas, R. L. 1976. Computing diagonal elements and inverse of a large numerator relationship matrix. Biometrics 32:949-953.

Robertson, A., and J. M. Rendel. 1954. The performance of heifers got by artificial insemination. J. Agric. Sci. 44:184-192.

Willham, R. L. 1968. New goals in undergraduate teaching of genetics. J. Anim. Sci. 27:888-892. 